\title{
Sex Differences in HIV Testing - 20 PEPFAR-Supported Sub-Saharan African Countries, 2019
}

Bakary Drammeh, DrPH ${ }^{1}$; Amy Medley, $\mathrm{PhD}^{1}$; Helen Dale, BVSc, MBChB ${ }^{1}$; Anindya K. De, $\mathrm{PhD}^{1}$; Shane Diekman, PhD ${ }^{1}$; Randy Yee, MSc ${ }^{1}$; Tiffiany Aholou, $\mathrm{PhD}^{1}$; Arielle Lasry, $\mathrm{PhD}^{1}$; Andrew Auld, $\mathrm{MD}^{2}$; Brittney Baack, MPH${ }^{2}$; Wayne Duffus, MD, $\mathrm{PhD}^{1}$; Ebrahim Shahul, MD ${ }^{1}$; Vincent Wong,

$\mathrm{MSc}^{3}$; Michael Grillo, $\mathrm{PhD}^{4}$; Teeb Al-Samarrai, $\mathrm{MD}^{5}$; Shabeen Ally, PhD ${ }^{5}$; Mtemwa Nyangulu, MCHD ${ }^{2}$; Rose Nyirenda, MD ${ }^{6}$; Jacobus Olivier, PhD;

Thato Chidarikire, $\mathrm{PhD}^{8}$; Nompumelelo Khanyile, $\mathrm{MBA}^{9}$; Alick A. Kayange, $\mathrm{MD}^{10}$; Oscar Ernest Rwabiyago, $\mathrm{MD}^{11}$; Upendo Kategile, MSc ${ }^{12}$; Jema Bisimba, MPH ${ }^{12}$; Rachel A. Weber, PhD ${ }^{13}$; Gertrude Ncube, $\mathrm{MPH}^{14}$; Onesimo Maguwu, MPH ${ }^{15}$; Ismelda Pietersen, MPH ${ }^{16}$; Denis Mali, MD ${ }^{17}$;

Edington Dzinotyiweyi, MA ${ }^{18}$; Lisa Nelson, MD ${ }^{19}$; Matovu John Bosco, MPH ${ }^{20}$; Kwarisiima Dalsone, MD ${ }^{21}$; Madina Apolot, MSc ${ }^{19}$; Samson Anangwe, $\mathrm{MSc}^{22}$; Leonard K. Soo, MPH${ }^{23}$; Mary Mugambi, MD24; Andre Mbayiha, MD ${ }^{25}$; Placidie Mugwaneza, MPH26; Samuel S. Malamba, PhD 27; Arlene Phiri, MCD ${ }^{28}$; Tina Chisenga, MD ${ }^{29}$; Mary Boyd, MD ${ }^{30}$; Chanie Temesgan, MD ${ }^{31}$; Mesfin Shimelis, MD ${ }^{31}$; Teklu Weldegebreal, MD ${ }^{32}$; Mirtie Getachew, MPH ${ }^{33}$; Shirish Balachandra, MD ${ }^{34}$; Ehui Eboi, PhD 35 ; Willibord Shasha, MD ${ }^{36}$; Nicole Doumatey, MSc ${ }^{34}$; Didier Adjoua, MD ${ }^{37}$; Chidozie Meribe, MD ${ }^{38}$; Jerry Gwamna, MD ${ }^{38}$; Pamela Gado, MPH ${ }^{39}$; Ima John-Dada, MSc ${ }^{40}$; Elie Mukinda, MD ${ }^{41}$; Luc F. Kamanga Lukusa, MD ${ }^{42}$; Lucien Kalenga, MD ${ }^{43}$; Sudhir Bunga, MD ${ }^{44}$; Victoria Achyut, MD ${ }^{45}$; John Mondi, MSc ${ }^{44}$; Peter Loeto, MSc ${ }^{46}$; Goabaone Mogomotsi, MPH ${ }^{47}$; Jenny Ledikwe, $\mathrm{PhD}^{48}$; Puleng Ramphalla, MSc ${ }^{49}$; Mphotleng Tlhomola, MSc ${ }^{50}$; Justine K. Mirembe, MBA ${ }^{51}$; Tse Nkwoh, MPH ${ }^{52}$; Laura Eno, MSc $\mathrm{c}^{52}$; Leonard Bonono, MSc $c^{53}$; Nely Honwana, MA ${ }^{54}$; Noela Chicuecue, MD ${ }^{55}$; Ana Simbine MSc ${ }^{56}$; Inacio Malimane, MD ${ }^{54}$; Lenhle Dube, BSc ${ }^{57}$; Munamato Mirira, MSc ${ }^{58}$; Phumzile Mndzebele, $\mathrm{MPH}^{59}$; Alean Frawley, $\mathrm{DO}^{60}$; Yolanda Marina Rebello Cardo, $\mathrm{MSc}^{60}$; Stephanie Behel, MPH ${ }^{1}$

Despite progress toward controlling the human immunodeficiency virus (HIV) epidemic, testing gaps remain, particularly among men and young persons in sub-Saharan Africa (1). This observational study used routinely collected programmatic data from 20 African countries reported to the U.S. President's Emergency Plan for AIDS Relief (PEPFAR) from October 2018 to September 2019 to assess HIV testing coverage and case finding among adults (defined as persons aged $\geq 15$ years). Indicators included number of HIV tests conducted, number of HIV-positive test results, and percentage positivity rate. Overall, the majority of countries reported higher HIV case finding among women than among men. However, a slightly higher percentage positivity was recorded among men (4.7\%) than among women (4.1\%). Provider-initiated counseling and testing (PITC) in health facilities identified approximately two thirds of all new cases, but index testing had the highest percentage positivity in all countries among both sexes. Yields from voluntary counseling and testing (VCT) and mobile testing varied by sex and by country. These findings highlight the need to identify and implement the most efficient strategies for HIV case finding in these countries to close coverage

Continuing Education examination available at https://www.cdc.gov/mmwr/mmwr_continuingEducation.html

\section{INSIDE}

1807 Multidisciplinary Community-Based Investigation of a COVID-19 Outbreak Among Marshallese and Hispanic/Latino Communities - Benton and Washington Counties, Arkansas, March-June 2020

1812 Disproportionate Incidence of COVID-19 Infection, Hospitalizations, and Deaths Among Persons Identifying as Hispanic or Latino - Denver, Colorado March-October 2020

1817 Regional Analysis of Coccidioidomycosis Incidence - California, 2000-2018

1822 Survey of Teen Noise Exposure and Efforts to Protect Hearing at School — United States, 2020

1827 Increase in Hospital-Acquired CarbapenemResistant Acinetobacter baumannii Infection and Colonization in an Acute Care Hospital During a Surge in COVID-19 Admissions - New Jersey, February-July 2020

1832 Notes from the Field: Interpretation of Rapid Diagnostic Tests for Leptospirosis During a Dengue Outbreak - Yap State, Federated States of Micronesia, 2019

1838 QuickStats

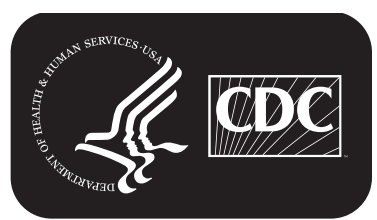


gaps. Strategies might need to be tailored for men who remain underrepresented in the majority of HIV testing programs.

In 2014, the Joint United Nations Programme on AIDS (UNAIDS) launched its 90-90-90 strategy for ending the global HIV pandemic: $90 \%$ of all persons living with HIV/AIDS (PLHIV) know their status; of these, $90 \%$ are receiving antiretroviral treatment (ART); and of these, $90 \%$ are virally suppressed (2). PEPFAR provides guidance on reaching these targets to all its supported countries (3). PEPFAR also collects data on standardized indicators as part of its Monitoring, Evaluation, and Reporting system (4). These data are collected by facility and community sites and are reported quarterly by each country program.

Routine program data reported to PEPFAR from October 2018 to September 2019 from 20 sub-Saharan African countries and modeling data from UNAIDS were used to identify progress toward achieving the first of the three 90-90-90 goals. These countries were selected because they collectively represent the highest HIV prevalence among PEPFAR-supported countries. Indicators used included the number of HIV tests conducted among adults, the number of HIV-positive test results, and yield (or percentage positivity) defined as the number of positive test results divided by the total number of tests reported. Results for each country are presented overall and disaggregated by sex and testing strategy. Testing strategies include index testing (offering an HIV test to the partners and biologic children of PLHIV), PITC (providers recommending an HIV test as part of routine care), VCT (HIV testing at a clinic dedicated to this purpose), and mobile testing (HIV testing offered at an ad hoc location in the community [e.g., van, workplace, or school]). The standard test statistic for the difference in proportions between men and women was computed on the basis of pooled variance formulation. P-values $<0.05$ were considered statistically significant for each pairwise difference and were computed under the asymptomatic normality assumption (5). PITC was used as the reference strategy for comparisons among strategies because the highest number of new HIV cases are identified by PITC in sub-Saharan Africa (1). The percentage positivity for the three other strategies (index testing, VCT, and mobile testing) was compared with PITC for each country and by sex.

From October 2018 to September 2019, PEPFAR supported 60,945,355 tests that identified 2,603,560 adults with positive HIV test results (5.0\% yield; Table 1 ). Approximately one fifth (19.9\%) of all testing occurred in South Africa. More women received tests than men (women, 40,263,510; men, $20,681,845)$. However, yield was slightly higher among men $(970,100 ; 4.7 \%$ yield) than among women $(1,633,460 ; 4.1 \%$ yield). Over one half (51.6\%) of all HIV-positive results among men were reported by South Africa, Tanzania, and Zambia, and approximately one third (29.2\%) were reported by South Africa alone.

Across the 19 countries (excluding Malawi because of limited data), PITC identified the most PLHIV (63.2\%) compared with index testing (17.4\%), VCT (11.0\%), and mobile testing (8.4\%). HIV case finding among men followed a similar

The MMWR series of publications is published by the Center for Surveillance, Epidemiology, and Laboratory Services, Centers for Disease Control and Prevention (CDC), U.S. Department of Health and Human Services, Atlanta, GA 30329-4027.

Suggested citation: [Author names; first three, then et al., if more than six.] [Report title]. MMWR Morb Mortal Wkly Rep 2020;69:[inclusive page numbers].

Centers for Disease Control and Prevention
Robert R. Redfield, MD, Director
Anne Schuchat, MD, Principal Deputy Director
Ileana Arias, PhD, Acting Deputy Director for Public Health Science and Surveillance
Rebecca Bunnell, PhD, MEd, Director, Office of Science
Jennifer Layden, MD, PhD, Deputy Director, Office of Science
Michael F. Iademarco, MD, MPH, Director, Center for Surveillance, Epidemiology, and Laboratory Services

MMWR Editorial and Production Staff (Weekly)

Charlotte K. Kent, PhD, MPH, Editor in Chief Jacqueline Gindler, MD, Editor

Paul Z. Siegel, MD, MPH, Guest Associate Editor Mary Dott, MD, MPH, Online Editor

Emilio Dirlikov, PhD, Guest Associate Online Editor Terisa F. Rutledge, Managing Editor

Douglas W. Weatherwax, Lead Technical Writer-Editor Glenn Damon, Soumya Dunworth, PhD,

Teresa M. Hood, MS, Jeffrey D. Sokolow, MA, Technical Writer-Editors

Matthew L. Boulton, MD, MPH

Carolyn Brooks, ScD, MA Jay C. Butler, MD

Virginia A. Caine, MD

Jonathan E. Fielding, MD, MPH, MBA

David W. Fleming, MD
Martha F. Boyd, Lead Visual Information Specialist Alexander J. Gottardy, Maureen A. Leahy,

Julia C. Martinroe, Stephen R. Spriggs, Tong Yang, Visual Information Specialists

Quang M. Doan, MBA, Phyllis H. King,

Terraye M. Starr, Moua Yang,

Information Technology Specialists
Ian Branam, MA, Acting Lead

Health Communication Specialist Shelton Bartley, MPH,

Lowery Johnson, Amanda Ray,

Jacqueline N. Sanchez, MS,

Health Communication Specialist Will Yang, MA,

Visual Information Specialist
MMWR Editorial Board

Timothy F. Jones, MD, Chairman Kate Galatas, MPH

William E. Halperin, MD, DrPH, MPH

Jewel Mullen, MD, MPH, MPA

Jeff Niederdeppe, $\mathrm{PhD}$

Celeste Philip, MD, MPH

Patricia Quinlisk, MD, MPH
Patrick L. Remington, MD, MPH

Carlos Roig, MS, MA

William Schaffner, MD

Nathaniel Smith, MD, MPH

Morgan Bobb Swanson, BS 


\begin{tabular}{|c|c|c|c|c|c|c|}
\hline \multirow[b]{3}{*}{ Country } & \multirow[b]{3}{*}{ All } & \multicolumn{2}{|c|}{ HIV tests conducted } & \multirow[b]{3}{*}{ All } & \multicolumn{2}{|c|}{ HIV tests positive } \\
\hline & & \multicolumn{2}{|c|}{ No. } & & \multicolumn{2}{|c|}{ No. (\%) } \\
\hline & & Men & Women & & Men & Women \\
\hline Rwanda & 888,336 & 371,405 & 516,931 & $7,343(0.8)$ & $2,929(0.8)$ & $4,414(0.9)$ \\
\hline Eswatini & 305,714 & 106,448 & 199,266 & $21,341(7.0)$ & $8,697(8.2)$ & $12,644(6.3)$ \\
\hline Botswana & 278,908 & 119,530 & 159,378 & $14,407(5.2)$ & $6,105(5.1)$ & $8,302(5.2)$ \\
\hline Namibia & 398,722 & 130,566 & 268,156 & 14,078 (3.5) & $5,385(4.1)$ & $8,693(3.2)$ \\
\hline Malawi & $3,741,494$ & $1,362,235$ & $2,379,259$ & $122,509(3.3)$ & $52,870(3.9)$ & $69,639(2.9)$ \\
\hline South Africa & $12,131,042$ & $3,996,848$ & $8,134,194$ & $759,465(6.3)$ & $267,255(6.7)$ & $492,210(6.1)$ \\
\hline Zimbabwe & $2,059,970$ & 709,379 & $1,350,591$ & $112,605(5.5)$ & $43,340(6.1)$ & $69,265(5.1)$ \\
\hline Kenya & $9,325,119$ & $3,248,359$ & $6,076,760$ & $168,809(1.8)$ & $60,515(1.9)$ & $108,294(1.8)$ \\
\hline Zambia & $4,666,548$ & $1,843,640$ & $2,822,908$ & 275,966 (5.9) & $111,599(6.1)$ & $164,367(5.8)$ \\
\hline Lesotho & 739,505 & 247,653 & 491,852 & 28,899 (3.9) & $11,453(4.6)$ & $17,446(3.5)$ \\
\hline Uganda & $4,872,644$ & $1,858,346$ & $3,014,298$ & $161,742(3.3)$ & $60,855(3.3)$ & $100,887(3.3)$ \\
\hline Ethiopia & 401,572 & 153,500 & 248,072 & $8,729(2.2)$ & $3,543(2.3)$ & $5,186(2.1)$ \\
\hline Tanzania & $6,930,758$ & $2,415,017$ & $4,515,741$ & $314,364(4.5)$ & $121,603(5.0)$ & $192,761(4.3)$ \\
\hline Cameroon & 839,762 & 317,881 & 521,881 & 32,435 (3.9) & 11,648 (3.7) & $20,787(4.0)$ \\
\hline Mozambique & $5,651,254$ & $1,519,954$ & $4,131,300$ & $281,022(5.0)$ & $103,433(6.8)$ & $177,589(4.3)$ \\
\hline Nigeria & $4,309,213$ & $1,348,056$ & $2,961,157$ & $158,351(3.7)$ & $55,834(4.1)$ & $102,517(3.5)$ \\
\hline Côte d'Ivoire & $2,200,382$ & 564,945 & $1,635,437$ & $60,058(2.7)$ & $19,713(3.5)$ & $40,345(2.5)$ \\
\hline DRC & 811,233 & 235,984 & 575,249 & $41,898(5.2)$ & $16,062(6.8)$ & $25,836(4.5)$ \\
\hline Angola & 141,292 & 49,215 & 92,077 & $9,208(6.5)$ & 3,253 (6.6) & $5,955(6.5)$ \\
\hline South Sudan & 251,887 & 82,884 & 169,003 & $10,331(4.1)$ & $4,008(4.8)$ & $6,323(3.7)$ \\
\hline Total & $60,945,355$ & $20,681,845$ & $40,263,510$ & $2,603,560(5.0)$ & $970,100(4.7)$ & $1,633,460(4.1)$ \\
\hline
\end{tabular}

Source: PEPFAR Monitoring, Evaluation, and Reporting data for Accountability, Transparency, and Impact Monitoring database, October $2018-S e p t e m b e r 2019$. Abbreviations: DRC = Democratic Republic of Congo; PEPFAR = U.S. President's Emergency Plan for AIDS Relief.

* Nine of the 20 countries account for $90 \%$ of HIV prevalence: Kenya, Malawi, Mozambique, Nigeria, South Africa, Tanzania, Uganda, Zambia, and Zimbabwe. Six countries have achieved the first 90 target (Botswana, Eswatini, Kenya, Lesotho, Namibia, and South Africa), and knowledge of HIV status is $<70 \%$ in Angola (69\%), DRC (49\%), and South Sudan (24\%).

pattern: PITC identified $57.7 \%$ of HIV-positive men, followed by index testing $(21.4 \%)$, VCT $(11.9 \%)$, and mobile testing (9.0\%). Six countries accounted for $80.2 \%$ of HIV-positive men identified through PITC: South Africa $(220,940)$, Mozambique $(56,960)$, Tanzania $(52,574)$, Zambia $(38,991)$, Kenya, $(28,814)$, and Uganda $(26,421)$. Additional data for new HIV cases identified across the four HIV testing strategies both overall and by sex are provided (Table 2).

Although PITC identified more PLHIV than any other testing strategy, the percentage positivity of index testing was higher than PITC in all countries and for both sexes (Table 3). The number of PLHIV identified through index testing ranged from 500 in South Sudan (11.0\% yield) to 116,500 in Tanzania ( $21.4 \%$ yield) (Table 2$)$. The five countries that identified the most PLHIV through index testing were Uganda (34,585), Mozambique $(40,681)$, Kenya $(51,717)$, Zambia $(63,587)$, and Tanzania $(116,546)$, and yields varied from $17.6 \%$ (Kenya) to $28.6 \%$ (Zambia). These countries also accounted for two thirds $(69.7 \%)$ of all HIV-positive men identified through index testing: Uganda $(15,313)$, Mozambique $(19,064)$, Kenya $(22,259)$, Zambia $(28,383)$, and Tanzania $(52,040)$. The contribution of index testing to the total number of HIV-positive men identified in these five countries ranged from $18.1 \%$ (Mozambique) to $42.8 \%$ (Tanzania).
The yield for VCT among men was significantly higher than for PITC in eight countries (Angola, Cameroon, Côte d'Ivoire, Malawi, Mozambique, Uganda, Zambia, and Zimbabwe) but was lower in the Democratic Republic of Congo (DRC) (Table 3). Similarly, mobile testing had a significantly higher yield than PITC among men in Cameroon, Côte d'Ivoire, DRC, Malawi, Mozambique, Nigeria, Uganda, and Zimbabwe but a lower yield in Botswana, Lesotho, and South Africa. Among women, VCT had a significantly higher yield than PITC in all countries except Botswana, whereas mobile testing had a significantly higher yield than PITC in all but three countries (Botswana, South Africa, and Kenya).

These findings will help guide the Ministries of Health in selecting specific testing strategies to increase HIV-testing coverage. This will help identify persons living with HIV infection.

\section{Discussion}

PITC identified the largest number of PLHIV, but index testing was more efficient at finding PLHIV as evidenced by a higher positivity rate. Several factors, including the absolute number of PLHIV identified, testing yield, cost per diagnosis, and the ability to reach persons with undiagnosed HIV infection, can help identify the best HIV-testing approach (1).

Although an efficient testing strategy in terms of identifying PLHIV, index testing is resource-intensive (1). For large yields 
Morbidity and Mortality Weekly Report

TABLE 2. Number and percentage of human immunodeficiency virus (HIV)-positive adults Identified by four HIV-testing strategies, overall and by sex - 20 PEPFAR-supported countries, October 2018-September 2019*

\begin{tabular}{|c|c|c|c|c|c|c|c|c|c|c|c|c|}
\hline \multirow[b]{3}{*}{ Country } & \multicolumn{3}{|c|}{ Index testing } & \multicolumn{3}{|c|}{ Provider-initiated testing } & \multicolumn{3}{|c|}{ Voluntary counseling and testing } & \multicolumn{3}{|c|}{ Mobile testing } \\
\hline & All & Men & Women & All & Men & Women & All & Men & Women & All & Men & Women \\
\hline & $\begin{array}{c}\text { No./Total no. }{ }^{\dagger} \\
(\%)^{\S}\end{array}$ & $\begin{array}{c}\% \\
\text { Positive }\end{array}$ & $\begin{array}{c}\% \\
\text { Positive }\end{array}$ & $\begin{array}{c}\text { No./Total no. } \\
(\%)\end{array}$ & $\begin{array}{c}\% \\
\text { Positive }\end{array}$ & $\begin{array}{c}\% \\
\text { Positive }\end{array}$ & $\begin{array}{c}\text { No./Total no. } \\
(\%)\end{array}$ & $\begin{array}{c}\% \\
\text { Positive }\end{array}$ & $\begin{array}{c}\% \\
\text { Positive }\end{array}$ & $\begin{array}{c}\text { No./Total no. } \\
(\%)\end{array}$ & $\begin{array}{c}\% \\
\text { Positive }\end{array}$ & $\begin{array}{c}\% \\
\text { Positive }\end{array}$ \\
\hline Rwanda & $1.4 / 26.6(5.1)$ & 4.6 & 5.8 & $2.4 / 545.9(0.5)$ & 0.5 & 0.4 & $2.6 / 259.9(1.0)$ & 0.8 & 1.2 & $0.9 / 55.9(1.7)$ & 1.0 & 2.8 \\
\hline Eswatini & $3.5 / 13.9(25.5)$ & 25.6 & 25.4 & $11.7 / 214.9(5.5)$ & 6.6 & $5.0^{9}$ & $4.0 / 46.6(8.5)$ & 7.9 & 9.1 & $2.1 / 30.3(6.9)$ & 6.5 & 7.2 \\
\hline Botswana & $2.3 / 16.9(13.8)$ & 14.6 & 12.9 & $9.8 / 187.5(5.3)$ & 5.2 & 5.3 & $0.5 / 17.3(3.0)$ & 2.6 & 3.3 & $1.7 / 57.1(3.0)$ & 2.6 & 3.4 \\
\hline Namibia** & $2.6 / 16.8(15.6)$ & 15.7 & 15.5 & $10.1 / 347.8(2.9)$ & 3.3 & 2.7 & $1.3 / 32.6(3.9)$ & 3.8 & 4.0 & $0.07 / 1.5(4.5)$ & 4.4 & 4.6 \\
\hline Malawi ${ }^{\text {t† }}$ & $8.9 / 29.8(30.1)$ & 30.5 & 29.6 & $29.7 / 1,025.5(2.9)$ & 3.4 & $2.7^{\pi}$ & $17.5 / 317.5(5.5)$ & 4.9 & $6.1^{\pi}$ & $5.6 / 63.5(8.9)$ & 7.5 & $11.1^{\uparrow}$ \\
\hline South Africa & 28.5/92.4 (30.9) & 30.0 & $31.7^{\pi}$ & $647.8 / 10.2(6.3)$ & 6.9 & $6.0^{4}$ & $2.2 / 26.3(8.3)$ & 7.3 & $11.2^{\text {q }}$ & $80.9 / 1,747.6(4.6)$ & 4.2 & $4.9^{9}$ \\
\hline Zimbabwe & $23.8 / 73.8(32.2)$ & 34.5 & $30.2^{\pi}$ & $79.6 / 1,904.1(4.2)$ & 4.4 & 4.1 & $5.0 / 45.5(11.1)$ & 9.7 & 11.9 & $4.2 / 36.6(11.5)$ & 9.4 & $13.5^{\pi}$ \\
\hline Kenya & $51.7 / 294.4(17.6)$ & 14.5 & 21.0 ? & $91.7 / 7,379.6(1.2)$ & 1.2 & 1.2 & $23.7 / 1,361.9(1.7)$ & 1.4 & 2.0 ? & $1.8 / 289.1(0.6)$ & 0.4 & 0.7 \\
\hline Zambia & $63.6 / 222.2(28.6)$ & 26.4 & $30.7^{\pi}$ & $105.1 / 2,884.3(3.6)$ & 3.8 & 3.6 & $87.3 / 1,455.2(6.0)$ & 5.6 & $6.3^{\pi}$ & $19.9 / 104.8(19.0)$ & 15.7 & $22.0^{\pi}$ \\
\hline Lesotho & $4.2 / 17.3(24.3)$ & 25.4 & 23.3 & $20.2 / 597.0(3.4)$ & 4.3 & 3.0 ? & $0.4 / 7.8(4.8)$ & 3.3 & 5.7 & $4.1 / 117.4(3.5)$ & 2.7 & $4.5^{\pi}$ \\
\hline Uganda & $34.6 / 189.8(18.2)$ & 16.1 & $20.4 \pi$ & $80.3 / 3,231.7(2.5)$ & 2.4 & 2.5 & $33.9 / 1,070.8$ (3.2) & 2.9 & 3.4 & $12.9 / 380.4(3.4)$ & 3.1 & 3.7 \\
\hline Ethiopia & 2.8/26.1 (10.8) & 10.5 & 11.1 & 4.3/313.6 (1.4) & 1.5 & 1.3 & $0.9 / 40.8(2.3)$ & 1.8 & 2.4 & $0.7 / 21.0(3.2)$ & 2.5 & 3.9 \\
\hline Tanzania & $116.5 / 544.0(21.4)$ & 19.9 & 22.9 ? & $153.9 / 5,192.5(3.0)$ & 3.2 & $2.9^{9}$ & $5.0 / 103.5(4.9)$ & 3.8 & $5.8^{\pi}$ & $38.9 / 1,090.6(3.6)$ & 3.4 & 3.7 \\
\hline Cameroon & $5.3 / 46.3(11.5)$ & 9.5 & $14.3^{\pi}$ & $21.4 / 708.1(3.0)$ & 2.8 & 3.1 & $4.5 / 66.2(6.9)$ & 5.1 & $8.3^{\pi}$ & 1.1/19.1 (5.8) & 4.5 & 7.0 \\
\hline Mozambique & 40.7/151.3 (26.9) & 24.9 & 28.9 ? & $177.4 / 4,540.9$ (3.9) & 5.7 & $3.4^{\pi}$ & $54.3 / 863.6(6.3)$ & 6.1 & 6.4 & $8.6 / 95.5(9.0)$ & 7.2 & $11.6^{\pi}$ \\
\hline Nigeria & $25.1 / 103.5(24.3)$ & 19.5 & $30.6^{\pi}$ & $79.4 / 3,007.8(2.6)$ & 3.2 & $2.4^{\pi}$ & $38.1 / 947.0(4.0)$ & 3.3 & $4.6^{\pi}$ & $15.8 / 250.9(6.3)$ & 6.1 & 6.4 \\
\hline Côte d'Ivoire & $14.1 / 88.2(16.0)$ & 12.4 & 20.7 ף & $39.5 / 1,983.5(2.0)$ & 2.4 & $1.9^{\text {ी }}$ & $0.3 / 5.2(6.6)$ & 5.9 & 7.4 & $6.1 / 123.4(4.9)$ & 4.2 & 5.5 \\
\hline DRC & $9.3 / 29.7(31.4)$ & 30.4 & 32.6 & $23.8 / 656.3(3.6)$ & 5.1 & $3.2^{\pi}$ & $1.7 / 37.1(4.6)$ & 3.1 & $7.1^{9}$ & $7.0 / 88.1(8.0)$ & 6.3 & $9.4 \pi$ \\
\hline Angola & $0.7 / 2.0(37.3)$ & 29.8 & $44.3^{\pi}$ & $3.7 / 83.3(4.5)$ & 5.0 & 4.2 & $3.5 / 32.7(10.8)$ & 8.5 & $13.2^{9}$ & $1.2 / 23.2(5.2)$ & 4.5 & 5.4 \\
\hline South Sudan & $0.5 / 4.9(11.0)$ & 11.4 & 10.7 & $6.1 / 186.4(3.3)$ & 4.5 & $2.8 \pi$ & $2.9 / 48.9(6.0)$ & 5.1 & 6.9 & $0.7 / 11.7(6.4)$ & 4.1 & 8.7 \\
\hline Total & $\begin{array}{r}440.5 / \\
1,990.0(22.1)\end{array}$ & 20.1 & $24.3^{9}$ & $\begin{array}{r}1,598.2 / \\
45,255.4(3.5)\end{array}$ & 3.9 & $2.7^{\uparrow}$ & $\begin{array}{r}289.7 / \\
6,786.8(4.3)\end{array}$ & 3.8 & $4.6^{\pi}$ & $\begin{array}{r}214.4 / \\
4,607.9(4.7)\end{array}$ & 4.2 & $5.0^{9}$ \\
\hline
\end{tabular}

Source: PEPFAR Monitoring, Evaluation, and Reporting data for Accountability, Transparency, and Impact Monitoring database, October 2018-September 2019.

Abbreviations: DRC = Democratic Republic of Congo; PEPFAR = U.S. President's Emergency Plan for AIDS Relief.

* Breakdown by strategy for 19 countries (excluding Malawi because of limited data).

${ }^{\dagger}$ Number of HIV tests positive divided by the number of HIV tests conducted. Numbers are reported in thousands with one decimal place.

$\S \%$ indicates percent yield.

" Statistically significant difference between men and women at $p$-value $<0.05$. The $p$-values were calculated based on $z$-tests for differences in binomial proportions with pooled variance.

** Community index testing program yield of $22.6 \%$ overall across adults.

t+ Data are from 5.5 districts $(\mathrm{N}=28)$ in Malawi.

of persons with a new HIV diagnosis to be identified, index testing requires a massive, potentially expensive, scale-up ( $($ ). However, Kenya, Tanzania, Mozambique, and Uganda have achieved substantial scale-up, demonstrating the feasibility of index testing in sub-Saharan Africa. Although the majority of PLHIV in sub-Saharan Africa receive their diagnosis through PITC, findings from this analysis show that index testing is identifying new PLHIV in many countries.

Screening tools that use a combination of clinical and behavioral questions to identify persons most likely to be HIV-positive can increase the percentage positivity of PITC. However, these screening tools must be validated to ensure high sensitivity and good specificity to avoid missed opportunities for diagnosis (3).

These findings show that men are less likely to be tested for HIV than women and represented 37\% of HIV-positive results. Although women were twice as likely to be tested for HIV, approximately one third of new cases identified occurred among men. In all countries, women are routinely tested for HIV as part of antenatal testing, regardless of their clinical or behavioral risk factors. This might help explain why the number of tests conducted was higher among women compared with men, but the percent positivity rate was lower. PITC and index testing identified the most HIV-positive men, but mobile testing remains an important strategy to reach men who are unable or unwilling to attend health care facilities. Interventions that might improve coverage among men include flexible hours, male counselors, and integration of HIV testing into screening for other chronic conditions (7). Although not included in this analysis, HIV self-testing, directly offered within health facilities $(8)$ or distributed by sex or needle-sharing partners as part of index testing (9), might also be an efficient method for reaching men.

The findings in this report are subject to at least three limitations. First, cost data are lacking. Second, data quality varies across countries despite PEPFAR's monitoring and reporting guidance. Finally, PITC data include antenatal testing, which results in more testing among women. Testing antenatal women at lower risk also explains why the yield for PITC is lower for women than men, even though the yield is higher 
TABLE 3. Percentage positivity of four human immunodeficiency virus (HIV) testing strategies in identifying new HIV-positive adults, stratified by sex - 20 PEPFAR-supported countries, October 2018-September 2019*

\begin{tabular}{|c|c|c|c|c|c|c|c|c|}
\hline \multirow[b]{3}{*}{ Country } & \multicolumn{8}{|c|}{$\%$ Yield } \\
\hline & \multicolumn{4}{|c|}{ Men } & \multicolumn{4}{|c|}{ Women } \\
\hline & $\begin{array}{c}\text { PITC as } \\
\text { reference* }^{*}\end{array}$ & Index testing ${ }^{\dagger}$ & $\mathrm{VCT}^{\S}$ & Mobile testing? & $\begin{array}{l}\text { PITC as } \\
\text { reference }\end{array}$ & Index testing & VCT & Mobile testing \\
\hline Rwanda & 0.5 & $4.6^{* *}$ & 0.8 & 1.0 & 0.4 & $5.8^{* *}$ & 1.2 & $2.8^{* *}$ \\
\hline Eswatini & 6.6 & $25.6^{* *}$ & 7.9 & 6.5 & 5.0 & $25.4^{* *}$ & $9.1^{* *}$ & $7.2^{* *}$ \\
\hline Botswana & 5.2 & $14.6^{* *}$ & 2.6 & $2.6^{* *}$ & 5.3 & $12.9^{* *}$ & 3.3 & 3.4 \\
\hline Namibia & 3.3 & $15.7^{* *}$ & 3.8 & 4.4 & 2.7 & $15.5^{* *}$ & 4.0 & 4.6 \\
\hline Malawi & 3.4 & $30.5^{* *}$ & $4.9^{* *}$ & $7.6^{* *}$ & 2.7 & $29.6^{* *}$ & $6.1^{* *}$ & $11.1^{* *}$ \\
\hline South Africa & 6.9 & $30.0^{* *}$ & 7.3 & $4.2^{* *}$ & 6.0 & $31.7^{* *}$ & $11.2^{* *}$ & $4.9^{* *}$ \\
\hline Zimbabwe & 4.4 & $34.5^{* *}$ & $9.7^{* *}$ & $9.4^{* *}$ & 4.1 & $30.2^{* *}$ & $11.9^{* *}$ & $13.5^{* *}$ \\
\hline Kenya & 1.2 & $14.5^{* *}$ & 1.4 & 0.4 & 1.2 & $21.0^{* *}$ & $2.0^{* *}$ & 0.7 \\
\hline Zambia & 3.8 & $26.4^{* *}$ & $5.6^{* *}$ & $15.7^{* *}$ & 3.6 & $30.7^{* *}$ & $6.3^{* *}$ & $22.0^{* *}$ \\
\hline Lesotho & 4.3 & $25.4^{* *}$ & 3.3 & $2.7^{* *}$ & 3.0 & $23.3^{* *}$ & 5.7 & $4.5^{* *}$ \\
\hline Uganda & 2.4 & $16.1^{* *}$ & $2.9^{* *}$ & $3.1^{* * *}$ & 2.5 & $20.4^{* *}$ & $3.4^{* *}$ & $3.7^{* *}$ \\
\hline Ethiopia & 1.5 & $10.5^{* *}$ & 1.8 & 2.5 & 1.3 & $11.1^{* *}$ & 2.4 & $3.9 * *$ \\
\hline Tanzania & 3.2 & $19.9^{* *}$ & 3.8 & 3.4 & 2.9 & $22.9^{* *}$ & $5.8^{* *}$ & $3.7^{* *}$ \\
\hline Cameroon & 2.8 & $9.5^{* *}$ & $5.1^{* *}$ & 4.5 & 3.1 & $14.3^{* *}$ & $8.3^{* *}$ & $7.0^{* *}$ \\
\hline Mozambique & 5.7 & $24.9 * *$ & 6.1 & $7.2^{* *}$ & 3.4 & $28.9 * *$ & $6.4^{* *}$ & $11.6^{* *}$ \\
\hline Nigeria & 3.2 & $19.5^{* *}$ & 3.3 & $6.1^{* *}$ & 2.4 & $30.6^{* *}$ & $4.6^{* *}$ & $6.4^{* *}$ \\
\hline Côte d'Ivoire & 2.4 & $12.4^{* *}$ & $5.9 * *$ & $4.2^{* *}$ & 1.9 & $20.7^{* *}$ & $7.4^{* *}$ & $5.5^{* *}$ \\
\hline DRC & 5.1 & $30.4^{* *}$ & 3.1 & 6.3 & 3.2 & $32.6^{* *}$ & $7.1^{* *}$ & $9.4^{* *}$ \\
\hline Angola & 5.0 & $29.8^{* *}$ & $8.5^{* *}$ & 4.5 & 4.2 & $44.3^{* *}$ & $13.2^{* *}$ & 5.4 \\
\hline South Sudan & 4.5 & $11.4^{* *}$ & 5.1 & 4.1 & 2.8 & $10.7^{* *}$ & $6.9^{* *}$ & $8.7^{* *}$ \\
\hline
\end{tabular}

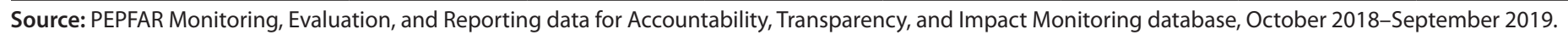

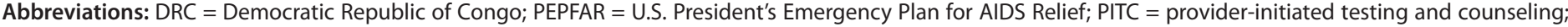
$\mathrm{VCT}=$ voluntary counseling and testing.

* PITC for HIV testing of persons at health facilities.

$\dagger$ Index testing of persons with new HIV diagnoses.

$\S$ VCT to determine HIV status.

ๆ Mobile testing outside health facilities.

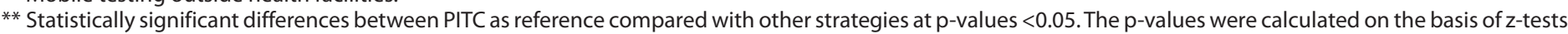
for differences in binomial proportions with pooled variance.

for women with the other three testing strategies. Strengths include the number of countries involved in the analysis and the volume of testing data collected by implementing partners.

As more PLHIV are identified, finding the remaining PLHIV will become more difficult and expensive because new cases will be harder to find. Multiple testing strategies, tailored to the epidemiologic context and targeted to populations with low access to HIV testing, can help reach these persons. Recency testing now allows programs to identify persons infected with HIV in the past year as well as clusters of incident cases. Immediate and intensified index testing efforts targeted to these clusters might reduce transmission and help countries achieve epidemic control (10).

Corresponding author: Bakary Drammeh, bdrammeh@cdc.gov, 404-718-8604.

\footnotetext{
${ }^{1}$ Division of Global HIV \& TB, Center for Global Health, CDC; ${ }^{2}$ Division
}

\section{Summary}

What is already known on this topic?

Identifying persons with HIV infection and initiating treatment are critical to ending the HIV epidemic by 2030. Despite global progress, testing gaps remain, particularly for men.

What is added by this report?

From October 2018 to September 2019, men in 20 sub-Saharan African countries were half as likely as women to receive an HIV test, and $37 \%$ of the HIV-positive results were among men. Similar sex differences were observed across HIV testing strategies based upon percent positivity rates.

What are the implications for public health practice?

These results highlight provider-initiated testing and index testing as strategies that might improve HIV testing coverage and maximize the number of persons newly identified as HIV-positive in 20 African countries. 
of Global HIV \& TB, Center for Global Health, CDC Malawi; ${ }^{3}$ US Agency for International Development, Office of HIV/AIDS, Washington, DC; ${ }^{4}$ US Department of Defense, HIV/AIDS Prevention Program; ${ }^{5}$ Office of the US Global AIDS Coordinator and Health Diplomacy, US Department of State; ${ }^{6}$ Ministry of Health, Malawi; ${ }^{7}$ Division of Global HIV \& TB, Center for Global Health, CDC South Africa; ${ }^{8}$ HIV Prevention Programmes, National Department of Health, Ministry of Health, South Africa; ${ }^{9}$ US Agency for International Development, South Africa; ${ }^{10}$ US Department of Defense, HIV/ AIDS Program, Tanzania; ${ }^{11}$ Division of Global HIV \& TB, Center for Global Health, CDC Tanzania; ${ }^{12}$ US Agency for International Development, Tanzania; ${ }^{13}$ Division of Global HIV \& TB, Center for Global Health, CDC Zimbabwe; ${ }^{14}$ Ministry of Health, Zimbabwe; ${ }^{15}$ US Agency for International Development, Zimbabwe; ${ }^{16}$ Division of Global HIV \& TB, Center for Global Health, CDC Namibia; ${ }^{17}$ US Agency for International Development, Namibia; ${ }^{18}$ Ministry of Health and Social Services, Namibia; ${ }^{19}$ Division of Global HIV \& TB, Center for Global Health, CDC Uganda; ${ }^{20}$ Ministry of Health, Uganda; ${ }^{21}$ US Agency for International Development Uganda; ${ }^{22}$ Department of Defence, Kenya; ${ }^{23}$ US Agency for International Development, Kenya; ${ }^{24}$ Ministry of Health, Kenya; ${ }^{25}$ Division of Global HIV \& TB, Center for Global Health, CDC Rwanda; ${ }^{26}$ Ministry of Health, Rwanda; ${ }^{27}$ Division of Global HIV \& TB, Center for Global Health, CDC Rwanda; ${ }^{28}$ US Agency for International Development, Zambia; ${ }^{29}$ Ministry of Health, Zambia; ${ }^{30}$ Division of Global HIV \& TB, Center for Global Health, CDC Zambia; ${ }^{31}$ Division of Global HIV \& TB, Center for Global Health, CDC Ethiopia; ${ }^{32}$ US Agency for International Development, Ethiopia; ${ }^{33}$ Federal Ministry of Health, Ethiopia; ${ }^{34}$ Division of Global HIV \& TB, Center for Global Health, CDC Côte D'Ivoire; ${ }^{35}$ Ministry of Health, Côte D'Ivoire; ${ }^{36}$ US Agency for International Development, Côte D'Ivoire; ${ }^{37}$ Department of Defense, Côte D'Ivoire; ${ }^{38}$ Division of Global HIV \& TB, Center for Global Health, CDC Nigeria; ${ }^{39}$ US Agency for International Development, Nigeria; ${ }^{40} \mathrm{Federal} \mathrm{Ministry} \mathrm{of}$ Health, Nigeria; ${ }^{41}$ Division of Global HIV \& TB, Center for Global Health, CDC Democratic Republic of Congo; ${ }^{42}$ Ministry of Health, Democratic Republic of Congo; ${ }^{43}$ US Agency for International Development, Democratic Republic of Congo; ${ }^{44}$ Division of Global HIV \& TB, Center for Global Health, CDC South Sudan; ${ }^{45}$ Ministry of Health, South Sudan; ${ }^{46}$ Division of Global HIV \& TB, Center for Global Health, CDC Botswana; ${ }^{47}$ Ministry of Health, Botswana; ${ }^{48}$ International Training and Education Center, Botswana; ${ }^{49}$ Division of Global HIV \& TB, Center for Global Health, CDC Lesotho; ${ }^{50}$ Ministry of Health, Lesotho; ${ }^{51}$ US Agency for International Development, Lesotho; ${ }^{2}$ Division of Global HIV \& TB, Center for Global Health, CDC Cameroon; ${ }^{53}$ Ministry of Health, Cameroon; ${ }^{54}$ Division of Global HIV \& TB, Center for Global Health, CDC Mozambique; ${ }^{55}$ Ministry of Health, Mozambique; ${ }^{56}$ US Agency for International Development, Mozambique; ${ }^{57}$ Ministry of Health, Eswatini; ${ }^{58}$ US Agency for International Development, Eswatini; ${ }^{59}$ Division of Global HIV \& TB, Center for Global Health, CDC Eswatini; ${ }^{60}$ Division of Global HIV \& TB, Center for Global Health, CDC Angola.

\section{References}

1. De Cock KM, Barker JL, Baggaley R, El Sadr WM. Where are the positives? HIV testing in sub-Saharan Africa in the era of test and treat. AIDS 2019;33:349-52. PMID:30557162 https://doi.org/10.1097/ QAD.0000000000002096

2. Joint United Nations Programme on HIV/AIDS (UNAIDS). 90-90-90: an ambitious treatment target to help end the AIDS Epidemic. Geneva, Switzerland: UNAIDS; 2014. https://www.unaids.org/sites/default/files/ media_asset/90-90-90_en.pdf

3. US President's Emergency Plan for AIDS Relief. PEPFAR 2020 country operational plan guidance for all PEPFAR countries. Washington, DC: US President's Emergency Plan for AIDS Relief; 2020. https://www. state.gov/wp-content/uploads/2020/01/COP20-Guidance_ Final-1-15-2020.pdf

4. US President's Emergency Plan for AIDS Relief. Monitoring, evaluation, and reporting indicator reference guide, version 2.4. Washington, DC: US President's Emergency Plan for AIDS Relief; 2019. https://www. state.gov/wp-content/uploads/2019/10/PEPFAR-MER-IndicatorReference-Guide-Version-2.4-FY20.pdf

5. Siegel and Castellan. Nonparametric statistics for the behavioral sciences. 2nd ed. New York, NY: McGraw-Hill; 1988.

6. Sharma M, Smith JA, Farquhar C, et al. Assisted partner notification services are cost-effective for decreasing HIV burden in western Kenya. AIDS 2018;32:233-41. PMID:29135576

7. Sharma M, Barnabas RV, Celum C. Community-based strategies to strengthen men's engagement in the HIV care cascade in sub-Saharan Africa. PLoS Med 2017;14:e1002262. PMID:28399122 https://doi. org/10.1371/journal.pmed.1002262

8. Dovel K, Shaba F, Offorjebe OA, et al. Effect of facility-based HIV self-testing on uptake of testing among outpatients in Malawi: a clusterrandomised trial. Lancet Glob Health 2020;8:e276-87. PMID:31981557 https://doi.org/10.1016/S2214-109X(19)30534-0

9. Choko AT, Corbett EL, Stallard N, et al. HIV self-testing alone or with additional interventions, including financial incentives, and linkage to care or prevention among male partners of antenatal care clinic attendees in Malawi: an adaptive multi-arm, multi-stage cluster randomised trial. PLoS Med 2019;16:e1002719. PMID:30601823 https://doi. org/10.1371/journal.pmed.1002719

10. Kim AA, Behel S, Northbrook S, Parekh BS. Tracking with recency assays to control the epidemic: real-time HIV surveillance and public health response. AIDS 2019;33:1527-9. PMID:31021850 https://doi. org/10.1097/QAD.0000000000002239 